\title{
Protective Role of Dietary Curcumin in the Prevention of the Oxidative Stress Induced by Chronic Alcohol with respect to Hepatic Injury and Antiatherogenic Markers
}

\author{
Ravi Varatharajalu, Mamatha Garige, Leslie C. Leckey, Karina Reyes-Gordillo, \\ Ruchi Shah, and M. Raj Lakshman
}

Lipid Research Laboratory, VA Medical Center and Department of Biochemistry and Molecular Medicine, The George Washington University, Washington, DC 20422, USA

Correspondence should be addressed to M. Raj Lakshman; raj.lakshman@va.gov

Received 18 June 2015; Revised 10 November 2015; Accepted 12 November 2015

Academic Editor: Silvana Hrelia

Copyright (C) 2016 Ravi Varatharajalu et al. This is an open access article distributed under the Creative Commons Attribution License, which permits unrestricted use, distribution, and reproduction in any medium, provided the original work is properly cited.

\begin{abstract}
Curcumin, an antioxidant compound found in Asian spices, was evaluated for its protective effects against ethanol-induced hepatosteatosis, liver injury, antiatherogenic markers, and antioxidant status in rats fed with Lieber-deCarli low menhaden (2.7\% of total calories from $\omega-3$ polyunsaturated fatty acids (PUFA)) and Lieber-deCarli high menhaden (13.8\% of total calories from $\omega$-3 PUFA) alcohol-liquid (5\%) diets supplemented with or without curcumin $(150 \mathrm{mg} / \mathrm{kg} / \mathrm{day})$ for 8 weeks. Treatment with curcumin protected against high $\omega$-3 PUFA and ethanol-induced hepatosteatosis and increase in liver injury markers, alanine aminotransferase, and aspartate aminotransferase. Curcumin upregulated paraoxonase 1 (PON1) mRNA and caused significant increase in serum PON1 and homocysteine thiolactonase activities as compared to high $\omega-3$ PUFA and ethanol group. Moreover, treatment with curcumin protected against ethanol-induced oxidative stress by increasing the antioxidant glutathione and decreasing the lipid peroxidation adduct 4-hydroxynonenal. These results strongly suggest that chronic ethanol in combination with high $\omega$-3 PUFA exacerbated hepatosteatosis and liver injury and adversely decreases antiatherogenic markers due to increased oxidative stress and depletion of glutathione. Curcumin supplementation significantly prevented these deleterious actions of chronic ethanol and high $\omega$-3 PUFA. Therefore, we conclude that curcumin may have therapeutic potential to protect against chronic alcohol-induced liver injury and atherosclerosis.
\end{abstract}

\section{Introduction}

Chronic alcohol consumption leads to alcoholic hepatosteatosis and consequently to inflammation, necrosis, fibrosis, and finally cirrhosis that afflicts millions worldwide and is one of the major causes of mortality in developed countries [1]. Liver alcohol dehydrogenase is primarily responsible for the oxidation of ethanol to acetaldehyde as well as hydroxyl radicals leading to the generation of reactive oxygen species (ROS). In addition, chronic ethanol-induced cytochrome P4502E1 (CYP2E1) mediated oxidation of ethanol also produces a state of oxidative stress by generating ROS within the cells including the $\alpha, \beta$-unsaturated aldehyde, 4 -hydroxy2-nonenal (4-HNE) that may be more harmful than ROS because it has a longer half-life and can easily diffuse into cellular membranes [2]. The 4-HNE, in turn, is likely to play a major role for the progression of alcoholic fatty liver and liver disease. Whereas high $\omega$-3 PUFA fish oil diet ( $14 \%$ of dietary calories from $\omega$-3 PUFA) causes severe hepatosteatosis liver injury [3], we showed that $\omega$-3 PUFA fish oil diet $(\sim 2.7 \%$ of dietary calories from $\omega$-3 PUFA) had protective effects [4].

Excess of ROS in alcoholics causes increased low density lipoproteins (LDL) oxidation that is avidly scavenged by macrophages leading to their transformation to foam cells that are atherogenic. Further, oxidized LDL is capable of stimulating endothelial cells to express adhesion molecules, which attract excess of foamy macrophages under the subendothelial layer, thereby promoting the seeding process of plaque formation. Incidence of atherosclerosis is high in chronic alcoholics due to higher level of ROS [5], homocysteine, 
and homocysteine thiolactone (HTL) [6]. Antiatherogenic enzyme, paraoxonase 1 (PON1), is predominantly synthesized in the liver and secreted into circulation, where it avidly binds and becomes an integral part of high density lipoproteins (HDL) [7]. Antiatherosclerotic role of PON1 is to inhibit the oxidation of LDL as well as to inactivate oxidized lipid molecules by hydrolyzing cholesteryl linoleate hydroxide (CL-OH) and cholesteryl linoleate hydroperoxide (CL-OOH) groups by its esterase activity, thus facilitating reverse cholesterol transport (RCT) from macrophages to the liver for degradation $[8,9]$. Other crucial function of PON1 is to hydrolyze highly toxic homocysteine thiolactone ultimately to methionine by methionine synthase pathway $[10,11]$.

A number of etiological agents of liver disease such as smoking, alcohol, high fat and high cholesterol, high polyunsaturated fatty acids (PUFA), lipopolysaccharide (LPS), iron, homocysteine, and HTL, ROS, and PON1 gene polymorphism adversely affect PON1 activity leading to cardiovascular disease (CVD) [11-18]. Selective antioxidants such as quercetin, pomegranate juice, vitamin $\mathrm{C}$ and folic acid, vitamins $\mathrm{C}$ and $\mathrm{E}$, resveratrol, and betaine have been shown to protect against CVD by restoring PON activity [11, 19-24].

Curcumin (diferuloylmethane), a derivative of the spice turmeric (Curcuma longa), has been used in traditional medicine for thousands of years [25]. It is known for its use in wound healing [26] and also as an antibacterial, antiviral, and antifungal agent [27]. Curcumin also has antioxidant and anti-inflammatory properties and prevents alcohol- or carbon tetrachloride-induced liver injury by inhibiting the activation of $\mathrm{NF} \kappa \mathrm{B}$ signaling cascade, and the subsequent induction of proinflammatory cytokines, as well as chemokines, cyclooxygenase-2 (COX-2), and inducible nitric oxide synthase (iNOS) in Kupffer cells $[28,29]$. A number of studies have shown that curcumin can reduce lipid peroxidation and liver and serum cholesterol in several liver injury models [30-32].

Therefore, in this present study, we have addressed for the first time the concerted protective role played by dietary curcumin on chronic ethanol and PUFA mediated adverse effects on hepatic liver injury due to oxidative stress as evidenced by alterations in liver (i) ROS, (ii) endogenous antioxidant, and reduced glutathione (GSH), as well as (iii) hepatic lipid score. In addition, we also evaluated the possible protective action of curcumin on the antiatherogenic gene, PON1/HTLase, as well as on plasma and liver lipids.

\section{Materials and Methods}

2.1. Animals. Female Wistar-Furth rats weighing between 130 and $150 \mathrm{~g}$ were purchased from Charles River Laboratories, Inc. (Wilmington, MA). Female animals were chosen because they are known to show more severe alcohol-induced liver injury than males [33-35]. They were housed in pairs per cage in plastic cages $(40 \times 24 \times 18 \mathrm{~cm})$, in a temperature-controlled room at $25^{\circ} \mathrm{C}$ with 12 -hour light: dark cycle. All rats were fed a pelleted commercial diet (Purina Rodent Chow, \#500, TMI Nutrition, St. Louis, MO) during the first week of acclimation
TABLE 1: Composition of various experimental diets, total fat, $\omega-3$ PUFA, and ethanol.

\begin{tabular}{lcccc}
\hline Dietary groups $(n=4)$ & \multicolumn{4}{c}{ As percent of total calories } \\
& Total Fat & Ethanol & Protein & Carbohydrate \\
\hline $\begin{array}{l}\text { Low } \omega \text {-3 PUFA control } \\
\text { (LFO) }\end{array}$ & $35^{*}$ & 0 & 18 & 47 \\
$\begin{array}{l}\text { Low } \omega \text {-3 PUFA + } \\
\text { ethanol (LFOE) }\end{array}$ & $35^{*}$ & 36 & 18 & 11 \\
$\begin{array}{l}\text { Low } \omega \text {-3 PUFA + ethanol } \\
+ \text { curcumin (LFOEC) }\end{array}$ & $35^{*}$ & 36 & 18 & 11 \\
\hline $\begin{array}{l}\text { High } \omega \text {-3 PUFA control } \\
\text { (HFO) }\end{array}$ & $35^{\dagger}$ & 0 & 18 & 47 \\
$\begin{array}{l}\text { High } \omega-3 \text { PUFA + } \\
\text { ethanol (HFOE) }\end{array}$ & $35^{\dagger}$ & 36 & 18 & 11 \\
$\begin{array}{l}\text { High } \omega \text {-3 PUFA }+ \\
\text { ethanol + curcumin } \\
\text { (HFOEC) }\end{array}$ & $35^{\dagger}$ & 36 & 18 & 11 \\
\hline
\end{tabular}

${ }^{*} 2.7 \%$ of total calories came from $\omega-3$ PUFA; ${ }^{\dagger} 13.8 \%$ of total calories came from $\omega$-3 PUFA.

TABLE 2: Composition of various diets according to individual ingredients.

\begin{tabular}{lcccc}
\hline \multirow{2}{*}{ Diet ingredient } & \multicolumn{4}{c}{ Composition, grams/L } \\
& LFO & LFOE & HFO & HFOE \\
\hline Casein & 41.4 & 41.4 & 41.4 & 41.4 \\
L-Cysteine & 0.5 & 0.5 & 0.5 & 0.5 \\
DL-Methionine & 0.3 & 0.3 & 0.3 & 0.3 \\
Menhaden oil & 8.5 & 8.5 & 39.6 & 39.6 \\
Olive oil & 28.4 & 28.4 & 0 & 0 \\
Safflower oil & 2.7 & 2.7 & 0 & 0 \\
Maltose dextrin & 115.2 & 25.6 & 115.2 & 25.6 \\
Cellulose & 10 & 10 & 10 & 10 \\
Mineral mix & 8.75 & 8.75 & 8.75 & 8.75 \\
Vitamin mix & 2.5 & 2.5 & 2.5 & 2.5 \\
Choline bitartrate & 0.53 & 0.53 & 0.53 & 0.53 \\
Xanthan gum & 3 & 3 & 3 & 3 \\
\hline
\end{tabular}

period after arrival. They were then divided randomly into the following low $\omega$-3 PUFA (LFO) and high $\omega$-3 PUFA (HFO) groups as depicted in Table 1. Each of the LFO $(n=12)$ and HFO ( $n=12)$ groups were further subdivided separately into three groups of 4 each: LFO or HFO (controls), LFO or HFO plus ethanol (LFOE or HFOE, 35\% of dietary calories derived from ethanol), and LFOE or HFOE supplemented with curcumin $150 \mathrm{mg} /$ day $/ \mathrm{kg}$ [36] body weight (LFOEC or HFOEC). All LFO and HFO diets were made isoenergetic by substituting ethanol calories with dextrin-maltose and all these groups were pair-fed for 8 weeks. The composition of each diet (Dyets Inc., Bethlehem, PA) according to individual ingredients is described in Table 2.

2.2. Lipid Analysis. Blood samples were collected and centrifuged at $3100 \mathrm{rpm}$ (Beckman $\mathrm{J} 6 \mathrm{M}$ ) for $10 \mathrm{~min}$ at $4^{\circ} \mathrm{C}$. Separated serum, plasma, and liver samples were frozen at $-80^{\circ} \mathrm{C}$ until assayed. Liver lipids were extracted as previously 
described [37]. Both plasma and liver lipids were determined using enzymatic reagents as described by the manufacturer (Teco Diagnostics, Anaheim, CA).

2.3. Quantification of Hepatosteatosis by Oil Red O. Livers from various experimental groups were cut into small pieces and washed immediately with ice cold PBS and mounted on optimum cutting temperature (OCT) embedding compound in peel-a-way embedding molds (Electron Microscope Sciences, Hatfield, PA). Liver tissues were cryosectioned and stained with oil red $\mathrm{O}$ to measure accumulation of lipid using an automated histometric system (Image-Pro Plus 6.1, Media Cybernetics, Bethesda, MD) as described previously [11]. The data are expressed as average oil red O percentage area of lipid staining. Values are means \pm SEM.

2.4. 4-Hydroxynonenal (4-HNE) Staining of Liver Sections. Liver tissue sections were dewaxed in xylene and rehydrated and antigen was retrieved in heat incubated citrate buffer for 3 minutes. After washing in Tris buffered saline (TBS/T) containing Tween $20(0.1 \%)$, sections were incubated overnight at $4^{\circ} \mathrm{C}$ in primary antibody raised in goat against 4-HNE (Millipore Incorporation, MA, USA) that was diluted to 1:100 in CAS-Block (Life Technologies Grand Island, NY) reagent. After an hour of secondary antibody incubation, a DAB Plus Substrate kit (Life Technologies) was used as the chromogen, and then slides were counterstained with hematoxylin. Intensity of 4-HNE-positive brown colored area was detected under optical microscopy (Observer Z1, Carl Zeiss Microimaging, Inc., Thornwood, NY) and \% of brown pixels were calculated using AxioVision Rel.4.8.2 software in 4 randomly selected microscopic fields (magnification 40x) per section of all groups $(n=4)$.

2.5. Serum Biomarkers of Liver Injury. Leakage of liver transaminases into the blood compartment was assessed by measuring serum alanine aminotransferase (ALT) and aspartate aminotransferase (AST) in each animal from the various experimental groups using commercial kits according to manufacturer's instructions (Teco Diagnostics, Anaheim, CA). Mean serum ALT and AST activity in each group are expressed as international units per liter (IU/L) \pm SEM.

2.6. RNA Isolation and Real-Time PCR. The total RNA was isolated from each liver using the Tri-Reagent (Molecular Research Center, Cincinnati, $\mathrm{OH}$ ) as per manufacturer's instructions. Isolated total RNA was reverse transcribed by in vitro transcription as described by the manufacturer (Invitrogen, Carlsbad, CA). Quantitative real-time PCR was performed using a Bio-Rad iCycler using the SYBR green PCR mix (Bio-Rad, Hercules, CA). To amplify the coding region of PON1 and $\beta$-actin, we used gene-specific primers for rat PON1 forward primer $5^{\prime}$-TGCTGGCTCACAAGATTCAC$3^{\prime}$ and reverse primer $5^{\prime}$-TTCCTTTGTACACAGCAGCG$3^{\prime}$ and $\beta$-actin (forward: $5^{\prime}$-GTCAGGTCACTATCGGC- ${ }^{\prime}$; reverse: $5^{\prime}$-CATGGATGCCACAGGATTCC-3'). Data were normalized to $\beta$-actin and then quantified.
2.7. Serum PON1 and HTLase Activity Measurement. Serum PON1 enzyme activity was determined with paraoxon (Sigma-Aldrich Inc., St. Louis, MO) as the substrate. PON1 activity was measured as described by us previously [11, 13]. One unit of international enzyme activity is equal to $1 \mathrm{nmol}$ of paraoxon hydrolyzed per min per mL serum. PON1 activity in each experimental group was expressed as percent of the activity in the corresponding control group.

Serum HTLase activity was measured using $10 \mathrm{mM} \gamma$ thiobutyrolactone (Sigma Inc.) as the substrate [11,38] and Ellman's procedure to monitor the accumulation of free

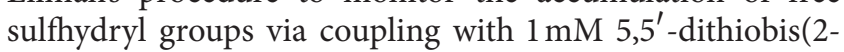
nitrobenzoic acid). The assay conditions were the same as for PON1 assay above and the rate of $\gamma$-thiobutyrolactone hydrolysis was expressed as nmoles per $\mathrm{mL}$ per min.

2.8. Liver Reduced Glutathione (GSH) Measurement. $1 \mathrm{~g}$ of tissue was weighed and washed with ice cold saline and homogenized in $10 \mathrm{~mL}$ of ice cold homogenizing buffer (10 mM Tris-HCl, pH 7.2; $250 \mathrm{mM}$ Sucrose; $1 \mathrm{mM}$ EDTA) containing $2 \mathrm{x}$ protease inhibitor cocktail. Reduced GSH was quantified according to manufacturer's instructions (Sigma Inc.).

2.9. Statistical Analysis. All data are presented as means \pm standard error of the mean (SEM). The data were analyzed as one-way ANOVA. This design was selected rather than the 3-way factorial design in order to save unnecessary animals in the curcumin treatment control groups. Pairwise comparisons and linear contrasts were not made where the overall ANOVA $p$ value exceeded 0.05 . In order to protect against inflation of the type 1 error rate, a Bonferroni adjustment (SAS Institute Inc., Cary, NC, USA) was made to the critical alpha value that was selected by dividing the 0.05 alpha by the number of preplanned comparisons. A $p$ value that was $\leq$ 0.05 was considered significant.

\section{Results}

3.1. Influence of Chronic Ethanol, Low w-3 PUFA, High w-3 PUFA, and Curcumin on Growth Pattern and Hepatosomatic Index in Various Experimental Groups. As shown in Table 3, animals from all experimental groups grew normally with significant gain in body weights, although the gains in the mean body weight in the ethanol fed groups were not as significant as in the corresponding control groups even when supplemented with curcumin. The mean liver weight relative to the mean body weight (hepatosomatic index) increased significantly by $23 \%(p<0.0001)$, in the high HFOE group but not in the LFOE group.

3.2. Influence of Chronic Ethanol, Low $\omega-3$ PUFA, High $\omega$ 3 PUFA, and Curcumin on the Status of Plasma and Liver Lipids. Table 4 shows that plasma triglycerides, VLDL-C, LDL-C, HDL-C, and total cholesterol were insignificantly altered in rats fed low PUFA alcohol group compared to control low PUFA group. LFOEC group showed significantly lowered plasma triglycerides compared to the LFOE group 
TABLE 3: Effect of ethanol and curcumin in low and high $\omega$-3 PUFA on liver weight to body weight ratio.

\begin{tabular}{lccccc}
\hline $\begin{array}{l}\text { Dietary groups } \\
(n=4)\end{array}$ & Initial body weight $(\mathrm{g})$ & Final body weight $(\mathrm{g})$ & Body weight gain $(\mathrm{g})$ & Final liver weight $(\mathrm{g})$ & Hepatosomatic index \\
\hline LFO & $130.1 \pm 3.9$ & $244.5 \pm 5.9$ & $114.4 \pm 6.2$ & $7.8 \pm 0.4$ & $3.2 \pm 0.16$ \\
LFOE & $160 \pm 4.8$ & $233.4 \pm 4.8$ & $73.4 \pm 4.8^{*}$ & $7.5 \pm 0.3$ & $3.2 \pm 0.20$ \\
LFOEC & $156.5 \pm 3.4$ & $209.0 \pm 4.4$ & $53 \pm 4.1^{\dagger}$ & $6.6 \pm 0.7^{\dagger}$ & $3.0 \pm 0.24$ \\
HFO & $159.4 \pm 2.9$ & $268.2 \pm 7.3$ & $108.8 \pm 4.3^{\S}$ & $8.4 \pm 0.2$ & $3.1 \pm 0.18$ \\
HFOE & $178.3 \pm 4.2$ & $228.3 \pm 6.7$ & $50.3 \pm 4.6^{*}$ & $8.8 \pm 0.3^{*}$ & $3.8 \pm 0.21^{*}$ \\
HFOEC & $157.4 \pm 3.3$ & $216.5 \pm 0.6$ & $59 \pm 2.8^{\ddagger}$ & $7.72 \pm 0.1^{\dagger}$ & $3.6 \pm 0.26^{*}$ \\
\hline
\end{tabular}

Values are means \pm SEM. Means in a column with different superscripts differ significantly $(p<0.05)$ as calculated by $t$-test with Bonferroni correction. ${ }^{*} p<0.0001$, compared to corresponding LFO or HFO groups; ${ }^{\dagger} p<0.0001$, compared to the corresponding LFOE or HFOE groups; ${ }^{\ddagger} p<0.0001$ compared to HFO; ${ }^{\S} p<0.02$ compared to LFO. Hepatosomatic index $=($ Liver weight $\times 100) /$ body weight.

TABLE 4: Effect of Chronic ethanol and curcumin on blood and livers lipids of rats fed low and high $\omega$-3 PUFA diets.

\begin{tabular}{|c|c|c|c|c|c|c|c|}
\hline \multirow{2}{*}{ Groups $n=4$} & \multicolumn{5}{|c|}{ Plasma (mg/dL) } & \multicolumn{2}{|c|}{ Liver $(\mathrm{mg} / 100 \mathrm{~g})$} \\
\hline & Triglycerides & VLDL-C & LDL-C & HDL-C & Total-C & Triglycerides & Total-C \\
\hline LFO & $83 \pm 5.5$ & $17 \pm 1.8$ & $24 \pm 2$ & $26 \pm 3$ & $66 \pm 8$ & $132 \pm 16$ & $56 \pm 6$ \\
\hline LFOE & $75 \pm 4.5^{*}$ & $15 \pm 1.7$ & $20 \pm 4$ & $25 \pm 6$ & $60 \pm 6$ & $146 \pm 14$ & $165 \pm 8^{*}$ \\
\hline LFOEC & $64 \pm 5^{\dagger}$ & $13 \pm 0.9$ & $15 \pm 3$ & $20 \pm 5$ & $48 \pm 7^{\dagger *}$ & $117 \pm 12$ & $117 \pm 12^{\dagger *}$ \\
\hline HFO & $104 \pm 8^{*}$ & $21 \pm 1.3^{*}$ & $6 \pm 0.6^{*}$ & $14 \pm 2^{\dagger *}$ & $40 \pm 7^{*}$ & $549 \pm 42^{*}$ & $201 \pm 8.0^{\dagger *}$ \\
\hline HFOE & $74 \pm 6.3^{\ddagger}$ & $15 \pm 1.5^{\ddagger}$ & $11 \pm 0.8^{\ddagger}$ & $21 \pm 4^{\ddagger}$ & $47 \pm 9$ & $734 \pm 49^{\ddagger}$ & $393 \pm 53^{\ddagger}$ \\
\hline HFOEC & $86 \pm 7^{\ddagger}$ & $17 \pm 2.0^{\ddagger}$ & $10 \pm 0.7^{\ddagger}$ & $14 \pm 2$ & $40 \pm 7$ & $633 \pm 55^{\dagger \neq}$ & $217 \pm 5.0^{\dagger \neq}$ \\
\hline
\end{tabular}

Values are means \pm SEM. Means in a column with different superscripts differ significantly $(p<0.05)$ as calculated by $t$-test with Bonferroni correction. ${ }^{*} p<$ 0.05 , compared to LFO; ${ }^{\dagger} p<0.05$, compared to the corresponding LFOE; ${ }^{\ddagger} p<0.05$ compared to the corresponding HFO.

$(p<0.002)$; however plasma triglycerides significantly increased in HFOEC group compared to HFOE group $(p<$ 0.04). HFOE group had significantly lower VLDL-C compared to HFO group $(p<0.0001)$. There was a tendency for plasma LDL-C, HDL-C, and total cholesterol to increase in HFOE group compared to HFOEC group, although the results were not statistically significant. Compared to respective control groups, both LFOE $(p<0.0001)$ and HFOE $(p<0.0005)$ groups exhibited significant increase in both liver triglycerides and cholesterol. Both liver triglycerides and cholesterol were significantly lower in LFOEC and HFOEC groups compared to the corresponding LFOE $(p<0.0001$, $p<0.0001)$ and HFOE $(p<0.0001, p<0.0001)$ groups, respectively.

3.3. Influence of Chronic Ethanol, Low w-3 PUFA, High w-3 PUFA, and Curcumin on Hepatosteatosis. As shown in Figures 1(a) and 1(b), histochemistry of oil red $O$ stained liver sections of low $\omega-3$ PUFA ethanol fed rats showed no significant changes of fat deposition compared to control. Ethanol significantly increased hepatosteatosis in rats fed high $\omega-3$ PUFA compared to other groups $(p<0.0001)$. Curcumin significantly reduced hepatosteatosis in HFOEC group compared to HFOE group $(p<0.002)$.

3.4. Influence of Chronic Ethanol, Low w-3 PUFA, High w-3 $P U F A$, and Curcumin on Liver Injury. As shown in Figures 2(a) and 2(b), serum alanine aminotransferase (ALT) and aspartate aminotransferase (AST) did not alter significantly between LFO and HFO groups. However, while serum AST and ALT increased significantly by $28 \%(p<0.008)$ and $40 \%$ $(p<0.008)$, respectively, in LFOE group compared to LFO group, these markers were significantly restored to the level of LFO group by curcumin supplementation ( $p<0.0002)$. Furthermore, serum ALT and AST increased dramatically by $60 \%(p<0.0001)$ and by $50 \%(p<0.0001)$, respectively, in HFOE compared to the HFO group. Curcumin feeding significantly blocked these dramatic increases in serum ALT and AST as compared to HFOE group $(p<0.0001)$.

3.5. Influence of Chronic Ethanol, Low $\omega-3$ PUFA, High $\omega-3$ PUFA, and Curcumin on Liver PON1 mRNA Expression. As shown in Figure 3, liver PON1 mRNA expression was decreased by $23 \%(p<0.01)$ in the high $\omega-3$ PUFA-fed group compared to the low $\omega-3$ PUFA group. Chronic ethanol feeding decreased liver PON1 mRNA expression by $25 \%$ $(p<0.001)$ and 30\% $(p<0.01)$ in both low and high $\omega-3$ PUFA groups, respectively, compared to low $\omega-3$ PUFA group. Curcumin significantly increased liver PON1 mRNA expression in high PUFA alcohol group $(p<0.04)$ but insignificantly increased PON1 mRNA in low PUFA alcohol groups.

3.6. Influence of Chronic Alcohol, Low w-3 PUFA, High w-3 PUFA, and Curcumin on Serum PON1 and HTLase Activity. As shown in Figure 4, high $\omega$-3 PUFA significantly decreased serum PON1 activity (Figure $4(\mathrm{a})$ ) by $20 \%(p<0.003)$ and HTLase activity (Figure $4(\mathrm{~b}))$ by $28 \%(p<0.0001$, 


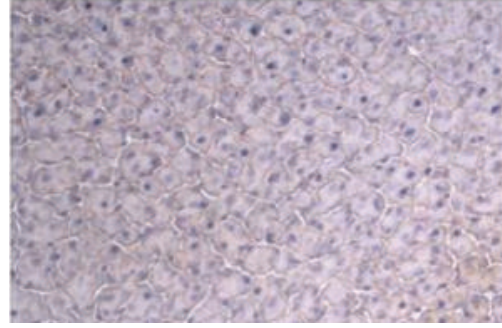

LFO

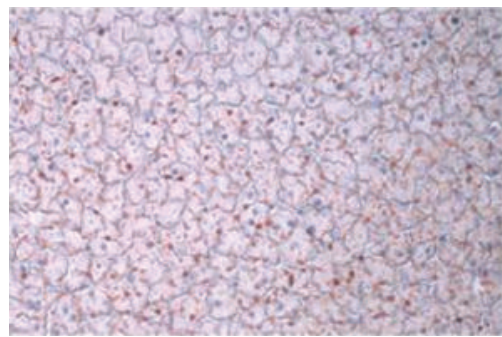

HFO

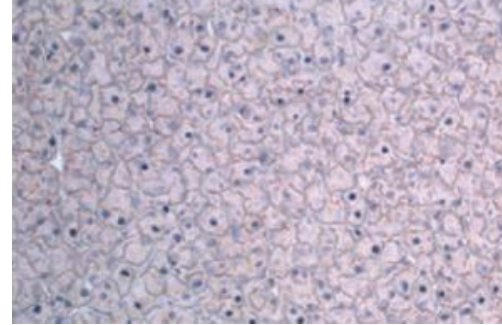

LFOE

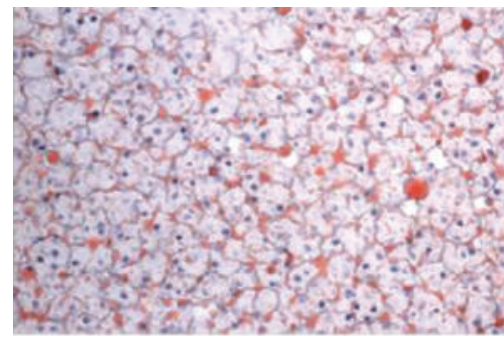

HFOE

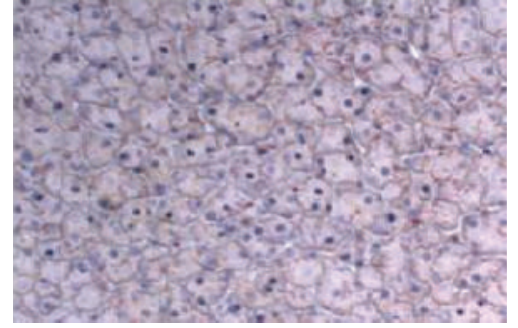

LFOEC

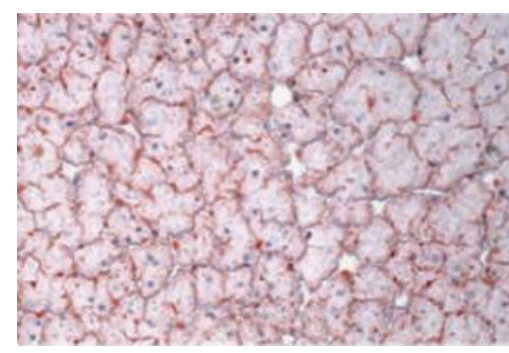

HFOEC

(a)

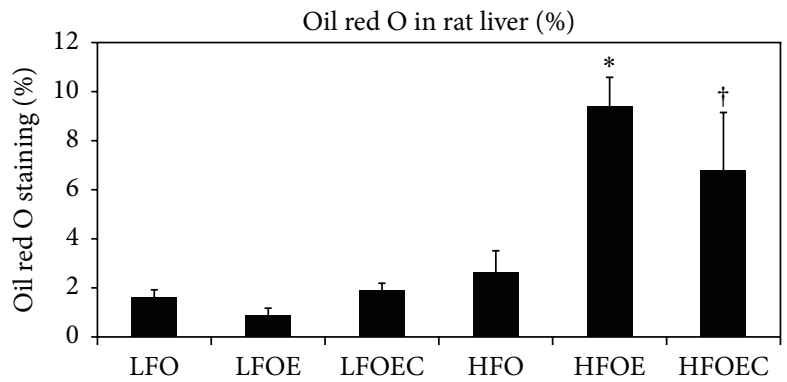

(b)

FIGURE 1: Histopathology of rat liver specimens and lipid deposits in the study groups. Cryosections of tissues were prepared as described in Section 2. Sections were stained with oil red $\mathrm{O}$ and counterstained with hematoxylin. (a) Representative 20x photomicrograph from the various groups. (b) Quantification of oil droplets in oil red O stained sections as determined by the Image-Pro Plus version $6.1 \mathrm{method}$. The data are expressed as average oil red O percentage area of lipid staining. Values are means \pm SEM. Statistical significance of variance was calculated using $t$-test with Bonferroni correction; ${ }^{*} p<0.0001$ compared to all groups except HFOEC group; ${ }^{\dagger} p<0.002$ compared to HFOE group.

Figure 4(b)) compared to low $\omega-3$ PUFA. Correspondingly, serum PON1 activity (Figure 4(a)) decreased by 23\% $(p<$ $0.05)$ in LFOE group and $58 \%(p<0.0001)$ in HFOE group while serum HTLase activity (Figure 4(b)) decreased by $25 \%(p<0.0001)$ and $59 \%(p<0.0001)$ in the low and high $\omega-3$ PUFA alcohol groups, respectively. Curcumin moderately stimulated serum PON1 activity by $15 \%(p<$ 0.05 ) (Figure $4(\mathrm{a})$ ) in the high $\omega$-3 PUFA alcohol group compared to HFOE group while it had no effect as compared to the LFOE group. Curcumin caused similar changes in serum HTLase activity only in the high $\omega$-3 PUFA ethanol group.

3.7. Influence of Chronic Ethanol, PUFA, and Curcumin on Liver GSH. As shown in Figure 5, there was no significant difference in the liver GSH levels in low or high $\omega$-3 PUFA fed groups. However, chronic ethanol significantly decreased liver GSH levels by $27 \%(p<0.0001)$ in low $\omega$-3 PUFA group and by $38 \%(p<0.005)$ in high $\omega-3$ PUFA group. Curcumin restored liver GSH levels in both low and high $\omega$-3 PUFA alcohol groups nearly to the control level $(p<0.05)$.

3.8. Influence of Chronic Ethanol, PUFA, and Curcumin on Lipid Peroxidation. To determine if the treatment with curcumin may have protected livers from the onset of alcoholinduced hepatitis, we determined levels of 4-HNE protein adduct which is an indicator of lipid peroxidation. Figure 6 shows the staining of 4 -HNE protein adducts was found to be significantly elevated in livers of LFOE (Figure 6(b)) and HFO (Figure 6(d)) and HFOE (Figure 6(e)) as compared to LFO (Figure 6(a)) group ( $p<0.0001)$. Curcumin feeding significantly lowered levels of 4-HNE protein adducts in both LFOEC (Figure 6(c)) and HFOEC (Figure 6(f)) as compared to LFOE and HFOE groups $(p<0.001)$, respectively.

\section{Discussion}

This study clearly demonstrates the ability of dietary curcumin to significantly attenuate chronic ethanol and $\omega$-3 PUFA 


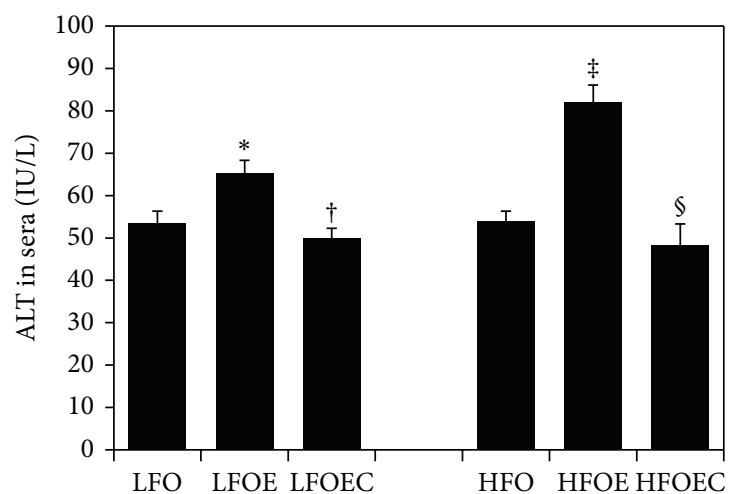

(a)

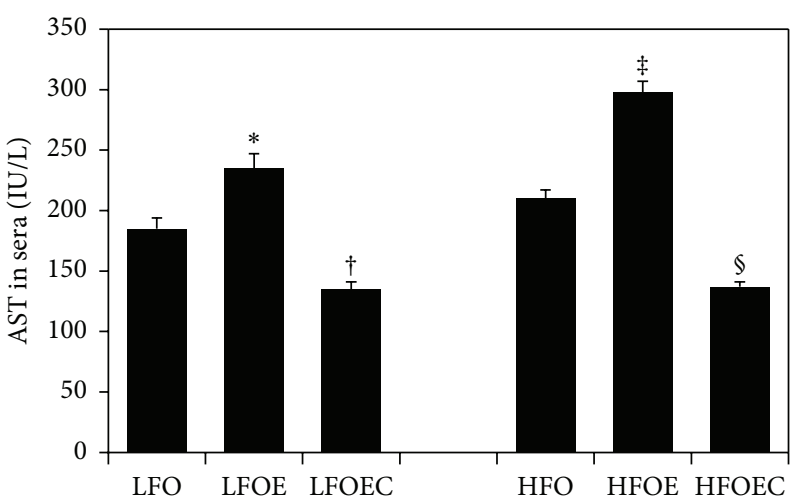

(b)

Figure 2: Influence of chronic ethanol, $\omega$-3 PUFA, and curcumin on serum ALT and AST in rats fed low and high $\omega$-3 PUFA diets. The animals in the indicated groups $(n=4)$ were pair-fed their respective Lieber-DeCarli alcohol containing liquid diets supplemented with the indicated concentration of curcumin for 8 weeks after which the animals were killed and each serum sample was analyzed. (a) ALT and (b) AST levels of these liver markers were measured as described in Section 2. The relative ALT and AST activities in the various experimental groups are expressed as international units per liter. The data are Means \pm SEM. Statistical significance of variance was calculated using $t$-test with Bonferroni correction; ${ }^{*} p<0.008$ compared to LFO group; ${ }^{\dagger} p<0.0002$ compared to the corresponding LFOE group; ${ }^{\ddagger} p<0.0001$ compared to HFO group; ${ }^{\S} p<0.0001$ compared to HFOE group.

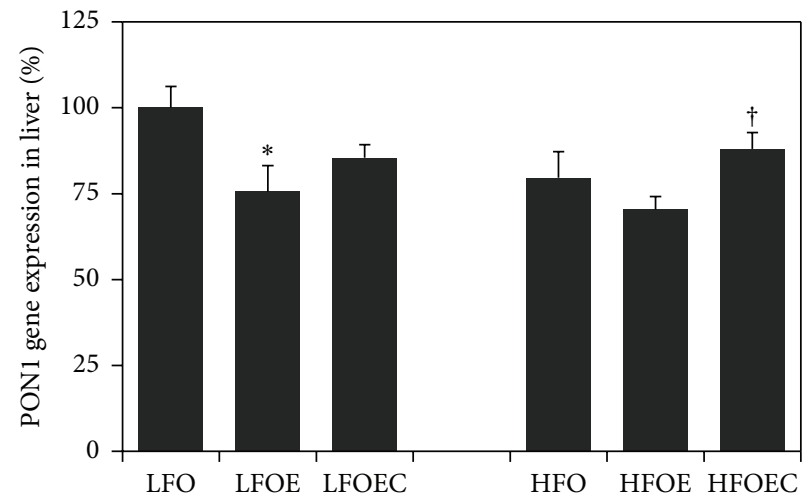

FIGURE 3: Effect of chronic ethanol and curcumin onPON1 mRNA expression in livers of rats fed low and high $\omega$-3 PUFA diets. The animals in the indicated groups $(n=4)$ were pair-fed their respective Lieber-DeCarli control or alcohol containing liquid diets for 8 weeks after which the animals were killed and each liver was analyzed for PON1 mRNA as described in Section 2. The relative expression of PON1 mRNA in the HFO group is expressed as percent of the corresponding values in the LFO group. The data are Means \pm SEM. Statistical significance of variance was calculated using $t$-test with Bonferroni correction; ${ }^{*} p<0.0001$ compared to LFO group; ${ }^{\dagger} p<0.04$ compared to HFOE group.

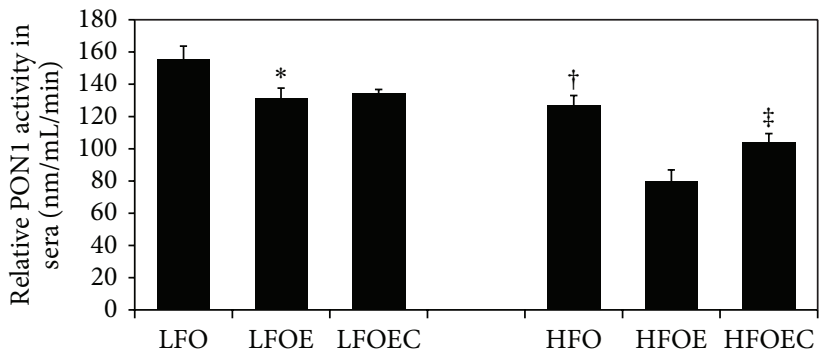

(a)

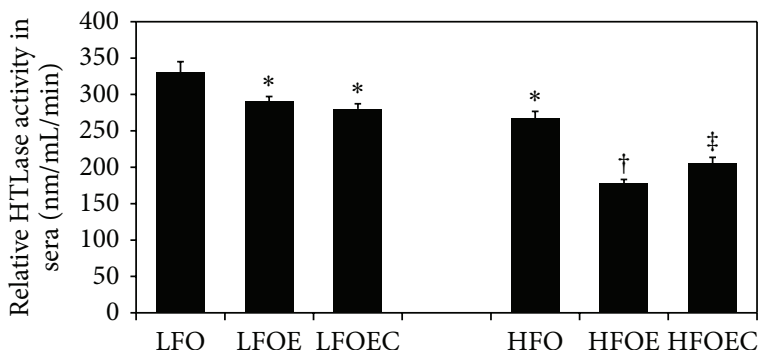

(b)

Figure 4: Influence of chronic ethanol and curcumin on serum PON1 and homocysteine thiolactonase activities were measured in rats fed low and high $\omega$-3 PUFA diets. The animals in the indicated groups $(n=4)$ were pair-fed their respective Lieber-DeCarli alcohol containing liquid diets supplemented with the indicated concentration of the curcumin for 8 weeks after which the animals were killed and serum sample was analyzed. (a) Serum PON1 activity measured using paraoxon as substrate. (b) Serum homocysteine thiolactonase activity was measured using $\gamma$-thiobutyrolactone as substrate. Enzyme activities in the various experimental groups are expressed as percent of the corresponding values in the LFO group. The data are Means \pm SEM. Statistical significance of variance was calculated using $t$-test with Bonferroni correction; ${ }^{*} p<0.0001$ compared to LFO group; ${ }^{\dagger} p<0.0001$ compared to LFO group; ${ }^{\ddagger} p<0.05$ compared HFOE group. 


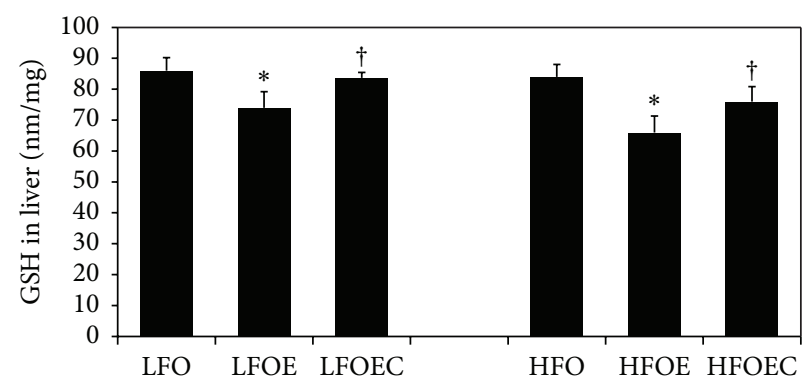

FIGURE 5: Influence of chronic ethanol, $\omega$-3 PUFA, and curcumin on liver GSH level in rats fed low and high $\omega$-3 PUFA diets. The animals in the indicated groups $(n=4)$ were pair-fed their respective Lieber-DeCarli alcohol containing liquid diets supplemented with the indicated concentration of curcumin for 8 weeks after which the animals were killed and each liver was analyzed for reduced GSH level in liver quantified as described in Section 2. The data are Means \pm SEM. Statistical significance of variance was calculated using $t$-test with Bonferroni correction; ${ }^{*} p<0.0001$ compared to corresponding LFO or HFO group; ${ }^{\dagger} p<0.005$ compared to the corresponding LFOE or HFOE group.

mediated hepatomegaly (Table 3), hyperlipidemia (Table 4), hepatosteatosis (Figure 1), steatohepatitis (Figure 2), and antiatherogenic markers. Furthermore, the protective properties of curcumin were associated with its antioxidant actions. This was assessed by biochemical and histological methods.

Consistent with numerous alcohol feeding studies, the present study confirms that alcohol-fed animals do not seem to gain as much weight as the control animals in spite of pairfeeding leading to increased hepatosomatic index (Table 3). Strikingly, curcumin feeding seems to further reduce the gain in body weight resulting in greater increase in the hepatosomatic index in both a low and a high PUFA alcohol diet, showing its ameliorative effects on hepatomegaly. These results may be related in part to the ability of curcumin to suppress adipogenesis.

The significant decreases in plasma triglycerides in LFOEC group compared to LFOE group (Table 4) could be explained due to the potential inhibitory action of curcumin on hepatic triglyceride and VLDL synthesis. This is reflected in significant decreases in plasma VLDL-C and total cholesterol. The present work clearly shows that feeding a high PUFA alcohol diet increased hepatosteatosis (measured with oil red $\mathrm{O}$ staining) and liver triglycerides and total cholesterol (Table 4), whereas curcumin feeding significantly prevented these effects on the hepatosteatotic action of chronic ethanol. These results are in accord with the effects of curcumin in ob/ob male mice fed high fat diet [39].

Levels of ALT and AST were increased in animals fed with high PUFA alcohol (Figure 2), indicating hepatocellular injury and dysfunction. It must be pointed out that the gross liver appearance and liver histopathology as well as ALT and AST data show that the liver injury caused by chronic ethanol is markedly alleviated by curcumin feeding in high $\omega$-3 fatty acid fed animals. Supporting our observations, curcumin has been shown to decrease hepatocellular injury and dysfunction in other hepatic injury models such as carbon tetrachloride [27, 36, 40], LPS/D-glucosamine [41], and methionine/choline deficiency [42].

Our present study clearly shows that feeding both low and high $\omega-3$ PUFA significantly downregulates the gene expression and activity of the multifunctional antioxidant enzyme PON1 in both total liver and serum (Figure 3).
These results are consistent with our previous study [11] and with those of Kudchodkar et al. [43]. Since chronic ethanol-induced cytochrome P4502E1 (Cyp2E1) exacerbates the accelerated generation of ROS in the presence of high $\omega$-3 PUFA, it is reasonable that this increased ROS may be responsible for these deleterious effects on PON1 status that is vulnerable to oxidative stress. The ability of curcumin to partially restore chronic ethanol and $\omega$-3 PUFA-mediated downregulation of serum PON1 activity and also PON1 mRNA in the liver as well as liver HTLase activity supports its antiatherogenic role of this antioxidant enzyme. Beneficial effects of other antioxidants such as pomegranate [44], vitamins $C$ and $E$ [22], and catechin [45] on serum PON1 activity are known. However, to our knowledge, this is the first time the protective effect of curcumin on chronic ethanol in combination with high $\omega-3$ PUFA mediated decrease in serum PON1 activity has been demonstrated. Since serum PON1 is an integral component of serum HDL-C there is a direct positive correlation between serum HDL-C and serum PON1 level. Therefore, the inability of curcumin to completely restore serum PON1 and HTLase activities in HFOEC group to their corresponding levels in HFO group may be due to the fact that curcumin also independently lowers plasma HDL-C (Table 4). It is well known that, apart from plasma HDL, PON1 is the only other most important antiatherogenic marker because of its ability to not only prevent LDL oxidation, but also destroy the other atherogenic molecule, homocysteine $[6-9,13-20]$.

The above antihepatosteatotic and antiatherogenic effects of curcumin are accompanied by reciprocal restoration of severely depleted liver GSH level and a reduction of 4-HNE lipid peroxidation product in chronic ethanol-fed group. These results are consistent with previous reports [27, 40] indicating that curcumin acts as an antioxidant. Our data are also consistent with previous studies, which demonstrated that curcumin induced de novo synthesis of GSH [46, 47]. These results strongly suggest the antioxidant capacity of curcumin in nullifying ethanol-induced ROS generation.

\section{Conclusion}

In conclusion, we have demonstrated that dietary curcumin not only effectively protects against the deleterious effects 


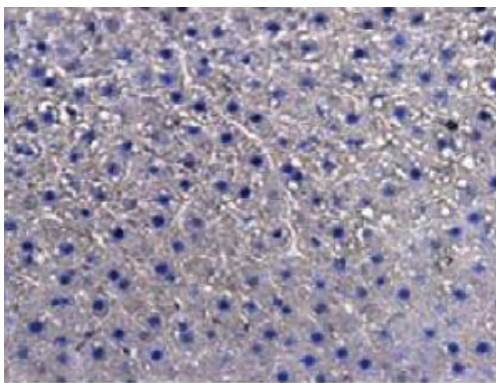

LFO

(a)

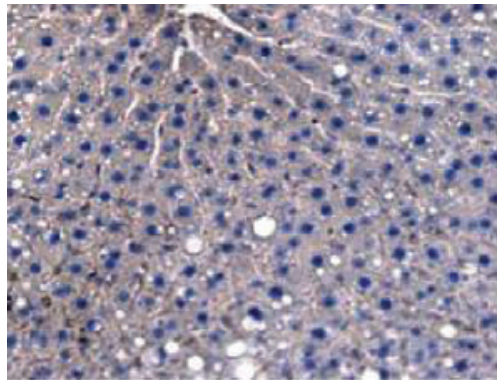

HFO

(d)

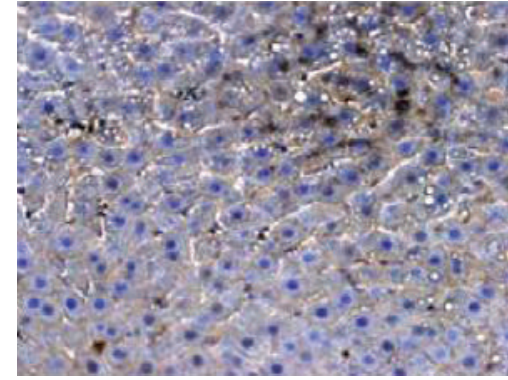

LFOE

(b)

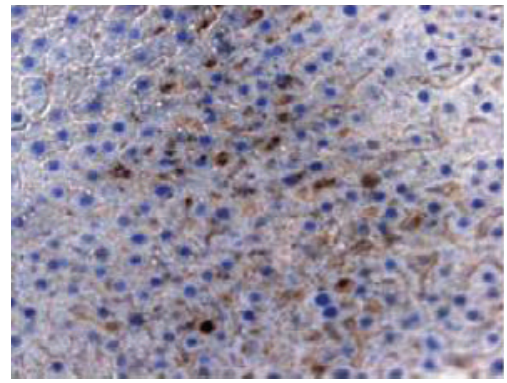

HFOE

(e)

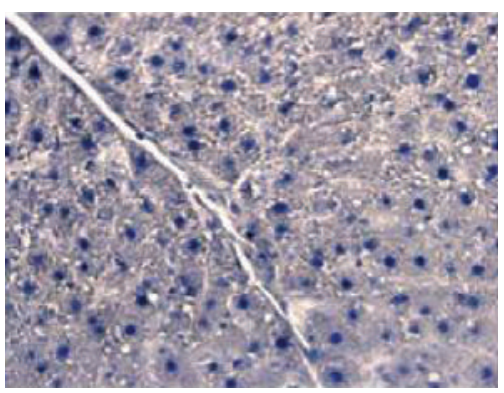

LFOEC

(c)

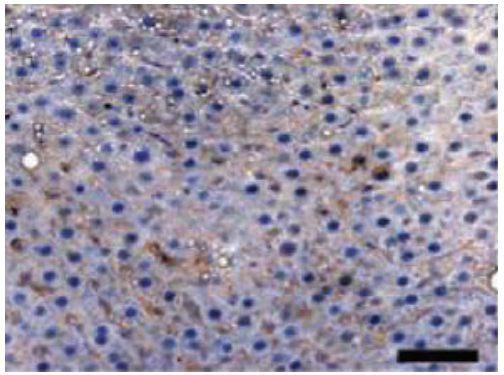

HFOEC

(f)

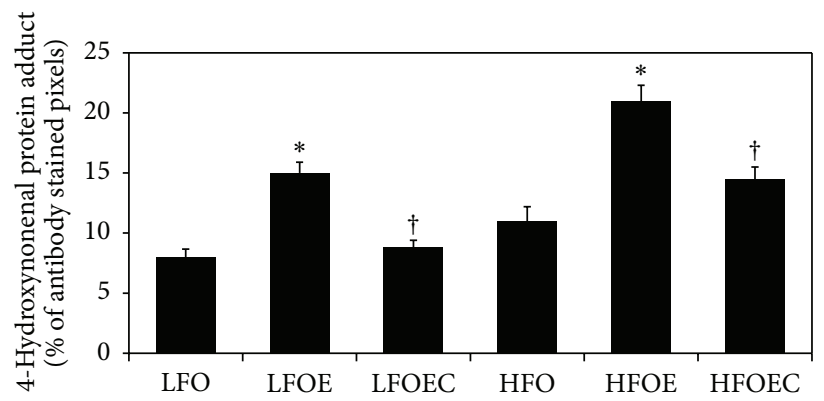

(g)

Figure 6: Effect of chronic alcohol, $\omega$-3 PUFA, and curcumin on 4-HNE protein adducts levels $(\times 200)$ in liver samples of various groups. The animals in the indicated groups $(n=4)$ were pair-fed their respective Lieber-DeCarli alcohol containing liquid diets supplemented with the indicated concentration of curcumin for 8 weeks after which the animals were killed and each rat liver sample was paraffin sectioned and stained with 4-HNE specific antibody. (1) Representative photomicrographs of immunohistochemically stained 4-HNE protein adducts (scale bars: $200 \mu \mathrm{m}$ ) in liver sections of following groups shown in (a) LFO; (b) LFOE; (c) LFOEC; (d) HFO; (e) HFOE; and (f) HFOEC and (g). Bar graph shows the \% of pixel values of brown color representing 4-HNE specific antibody reactivity from four randomly selected regions per section of each group $(n=4)$ measured using AxioVision Rel.4.8.2 software. The data are Means \pm SEM. Statistical significance of variance was calculated using $t$-test with Bonferroni correction; ${ }^{*} p<0.0001$ compared to all groups except HFOEC group; ${ }^{\dagger} p<0.001$ compared to HFOE group.

of chronic ethanol in combination with high PUFA on liver injury, but also favorably improves the antiatherogenic markers such as PON1/HTLase and GSH. These findings raise the possibilities that consuming traditional natural antioxidants compounds like curcumin may benefit health by modulating the lipid metabolism in alleviating hepatosteatosis and suppressing atherogenesis.

\section{Conflict of Interests}

The authors declare that there is no conflict of interests regarding the publication of this paper.

\section{Acknowledgments}

This work was supported partly by a Merit Grant from Department of Veterans Affairs and partly by NIH Grant R01AA0207020.

\section{References}

[1] P. E. Molina, C. McClain, D. Valla et al., "Molecular pathology and clinical aspects of alcohol-induced tissue injury," Alcoholism: Clinical and Experimental Research, vol. 26, no. 1, pp. 120-128, 2002. 
[2] H. Esterbauer, R. J. Schaur, and H. Zollner, "Chemistry and biochemistry of 4-hydroxynonenal, malondialdehyde and related aldehydes," Free Radical Biology and Medicine, vol. 11, no. 1, pp. 81-128, 1991.

[3] A. A. Nanji, S. Zhao, S. M. H. Sadrzadeh, A. J. Dannenberg, S. R. Tahan, and D. J. Waxman, "Markedly enhanced cytochrome P450 2E1 induction and lipid peroxidation is associated with severe liver injury in fish oil-ethanol-fed rats," Alcoholism: Clinical and Experimental Research, vol. 18, no. 5, pp.1280-1285, 1994.

[4] M. R. Lakshman, S. J. Chirtel, and L. L. Chambers, "Roles of omega 3 fatty acids and chronic ethanol in the regulation of plasma and liver lipids and plasma apoproteins A1 and E in rats," Journal of Nutrition, vol. 118, no. 11, pp. 1299-1303, 1988.

[5] W. Wu and A. I. Cederbaum, Alcohol, Oxidative Stress, and Free Radical Damage, NIAAA Publication, 2004.

[6] M. L. Cravo, L. M. Gloria, J. Selhub et al., "Hyperhomocysteinemia in chronic alcoholism: correlation with folate, vitamin B12, and vitamin B-6 status," The American Journal of Clinical Nutrition, vol. 63, no. 2, pp. 220-224, 1996.

[7] S. Deakin, I. Leviev, M. Gomaraschi, L. Calabresi, G. Franceschini, and R. W. James, "Enzymatically active paraoxonase-1 is located at the external membrane of producing cells and released by a high affinity, saturable, desorption mechanism," Journal of Biological Chemistry, vol. 277, no. 6, pp. 4301-4308, 2002.

[8] M. Aviram, M. Rosenblat, C. L. Bisgaier, R. S. Newton, S. L. Primo-Parmo, and B. N. La Du, "Paraoxonase inhibits highdensity lipoprotein oxidation and preserves its functions. A possible peroxidative role for paraoxonase," The Journal of Clinical Investigation, vol. 101, no. 8, pp. 1581-1590, 1998.

[9] O. Rozenberg, D. M. Shih, and M. Aviram, "Human serum paraoxonase 1 decreases macrophage cholesterol biosynthesis: possible role for its phospholipase- $\mathrm{A}_{2}$-like activity and lysophosphatidylcholine formation," Arteriosclerosis, Thrombosis, and Vascular Biology, vol. 23, no. 3, pp. 461-467, 2003.

[10] H. Jakubowski, "Calcium-dependent human serum homocysteine thiolactone hydrolase. A protective mechanism against protein N-homocysteinylation," The Journal of Biological Chemistry, vol. 275, no. 6, pp. 3957-3962, 2000.

[11] R. Varatharajalu, M. Garige, L. C. Leckey, M. Gong, and M. R. Lakshman, "Betaine protects chronic alcohol and $\omega-3$ PUFAmediated down-regulations of PON1 gene, serum PON1 and homocysteine thiolactonase activities with restoration of liver GSH," Alcoholism: Clinical and Experimental Research, vol. 34, no. 3, pp. 424-431, 2010.

[12] E. Nishio and Y. Watanabe, "Cigarette smoke extract inhibits plasma paraoxonase activity by modification of the enzyme's free thiols," Biochemical and Biophysical Research Communications, vol. 236, no. 2, pp. 289-293, 1997.

[13] M. N. Rao, P. Marmillot, M. Gong et al., "Light, but not heavy alcohol drinking, stimulates paraoxonase by up regulating liver mRNA in rats and humans," Metabolism, vol. 52, no. 10, pp. 1287-1294, 2003.

[14] I. Durak, H. Özbek, E. Devrim, N. Karagenç, and I. B. Ergüder, "Effects of cholesterol supplementation on antioxidant enzyme activities in rat hepatic tissues: possible implications of hepatic paraoxonase in atherogenesis," Nutrition, Metabolism and Cardiovascular Diseases, vol. 14, no. 4, pp. 211-214, 2004.

[15] L. G. Costa and C. E. Furlong, Eds., Paraoxonase (PON1) in Health and Disease: Basic and Clinical Aspects, Kluwer Academic Publishers, Norwell, Mass, USA, 2002.
[16] K. Trudel, D. Sinnett, R. W. James et al., "Iron-ascorbic acidinduced oxidant stress and its quenching by paraoxonase 1 in HDL and the liver: comparison between humans and rats," Journal of Cellular Biochemistry, vol. 96, no. 2, pp. 404-411, 2005.

[17] G. Ferretti, T. Bacchetti, E. Marotti, and G. Curatola, "Effect of homocysteinylation on human high-density lipoproteins: a correlation with paraoxonase activity," Metabolism, vol. 52, no. 2, pp. 146-151, 2003.

[18] M.-C. Blatter Garin, R. W. James, P. Dussoix et al., "Paraoxonase polymorphism Met-Leu54 is associated with modified serum concentrations of the enzyme. A possible link between the paraoxonase gene and increased risk of cardiovascular disease in diabetes," Journal of Clinical Investigation, vol. 99, no. 1, pp. 62-66, 1997.

[19] M. Gong, M. Garige, R. Varatharajalu et al., "Quercetin upregulates paraoxonase 1 gene expression with concomitant protection against LDL oxidation," Biochemical and Biophysical Research Communications, vol. 379, no. 4, pp. 1001-1004, 2009.

[20] M. Aviram, M. Rosenblat, D. Gaitini et al., "Pomegranate juice consumption for 3 years by patients with carotid artery stenosis reduces common carotid intima-media thickness, blood pressure and LDL oxidation," Clinical Nutrition, vol. 23, no. 3, pp. 423-433, 2004.

[21] M. F. Gursu, M. Onderci, F. Gulcu, and K. Sahin, "Effects of vitamin $\mathrm{C}$ and folic acid supplementation on serum paraoxonase activity and metabolites induced by heat stress in vivo," Nutrition Research, vol. 24, no. 2, pp. 157-164, 2004.

[22] G. P. Jarvik, N. T. Tsai, L. A. McKinstry et al., "Vitamin C and $\mathrm{E}$ intake is associated with increased paraoxonase activity," Arteriosclerosis, Thrombosis, and Vascular Biology, vol. 22, no. 8, pp. 1329-1333, 2002.

[23] B. F. Curtin, K. I. Seetharam, P. Dhoieam, R. K. Gordon, B. P. Doctor, and M. P. Nambiar, "Resveratrol induces catalytic bioscavenger paraoxonase 1 expression and protects against chemical warfare nerve agent toxicity in human cell lines," Journal of Cellular Biochemistry, vol. 103, no. 5, pp. 1524-1535, 2008.

[24] M. Garige, M. Gong, R. Varatharajalu, and M. R. Lakshman, "Quercetin up-regulates paraoxonase 1 gene expression via sterol regulatory element binding protein 2 that translocates from the endoplasmic reticulum to the nucleus where it specifically interacts with sterol responsive element-like sequence in paraoxonase 1 promoter in HuH7 liver cells," Metabolism, vol. 59, no. 9, pp. 1372-1378, 2010.

[25] S. Prasad and B. B. Aggarwal, "Turmeric, the golden spice: from traditional medicine to modern medicine," in Herbal Medicine: Biomolecular and Clinical Aspects, I. F. F. Benzie and S. WachtelGalor, Eds., chapter 13, CRC Press, Boca Raton, Fla, USA, 2nd edition, 2011.

[26] D. Akbik, M. Ghadiri, W. Chrzanowski, and R. Rohanizadeh, "Curcumin as a wound healing agent," Life Sciences, vol. 116, no. 1, pp. 1-7, 2014.

[27] S. Z. Moghadamtousi, H. Abdul Kadir, P. Hassandarvish, H. Tajik, S. Abubakar, and K. Zandi, "A review on antibacterial, antiviral, and antifungal activity of curcumin," BioMed Research International, vol. 2014, Article ID 186864, 12 pages, 2014.

[28] K. Reyes-Gordillo, J. Segovia, M. Shibayama, P. Vergara, M. G. Moreno, and P. Muriel, "Curcumin protects against acute liver damage in the rat by inhibiting NF- $\kappa \mathrm{B}$, proinflammatory cytokines production and oxidative stress," Biochimica et Biophysica Acta, vol. 1770, no. 6, pp. 989-996, 2007. 
[29] A. A. Nanji, K. Jokelainen, G. L. Tipoe, A. Rahemtulla, P. Thomas, and A. J. Dannenberg, "Curcumin prevents alcoholinduced liver disease in rats by inhibiting the expression of NF- $\kappa$ B-dependent genes," The American Journal of PhysiologyGastrointestinal and Liver Physiology, vol. 284, no. 2, pp. G321G327, 2003.

[30] H.-I. Lee, R. A. McGregor, M.-S. Choi et al., "Low doses of curcumin protect alcohol-induced liver damage by modulation of the alcohol metabolic pathway, CYP2E1 and AMPK," Life Sciences, vol. 93, no. 18-19, pp. 693-699, 2013.

[31] M. Y. Um, K. H. Hwang, J. Ahn, and T. Y. Ha, "Curcumin attenuates diet-induced hepatic steatosis by activating AMPactivated protein kinase," Basic and Clinical Pharmacology and Toxicology, vol. 113, no. 3, pp. 152-157, 2013.

[32] H. M. M. Arafa, "Curcumin attenuates diet-induced hypercholesterolemia in rats," Medical Science Monitor, vol. 11, no. 7, pp. BR228-BR234, 2005.

[33] H. Kono, M. D. Wheeler, I. Rusyn et al., "Gender differences in early alcohol-induced liver injury: role of $\mathrm{CD} 14, \mathrm{NF} \kappa \mathrm{B}$ and TNF $\alpha$," American Journal of Physiology-Gastrointestinal and Liver Physiology, vol. 278, pp. G652-G661, 2000.

[34] A. A. Nanji, K. Jokelainen, M. Fotouhinia et al., "Increased severity of alcoholic liver injury in female rats: role of oxidative stress, endotoxin, and chemokines," American Journal of Physiology-Gastrointestinal and Liver Physiology, vol. 281, no. 6, pp. G1348-G1356, 2002.

[35] S. D. Tadic, M. S. Elm, H.-S. Li et al., "Sex differences in hepatic gene expression in rat model of ethanol-induced liver injury," Journal of Applied Physiology, vol. 93, no. 3, pp. 1057-1068, 2002.

[36] E.-J. Park, C. H. Jeon, G. Ko, J. Kim, and D. H. Sohn, "Protective effect of curcumin in rat liver injury induced by carbon tetrachloride," Journal of Pharmacy and Pharmacology, vol. 52, no. 4, pp. 437-440, 2000.

[37] R. B. Shireman and J. Durieux, "Microplate methods for determination of serum cholesterol, high density lipoprotein cholesterol, triglyceride and apolipoproteins," Lipids, vol. 28, no. 2, pp. 151-155, 1993.

[38] T. Kosaka, M. Yamaguchi, T. Motomura, and K. Mizuno, "Investigation of the relationship between atherosclerosis and paraoxonase or homocysteine thiolactonase activity in patients with type 2 diabetes mellitus using commercially available assay," Clinica Chimica Acta, vol. 359, no. 1-2, pp. 156-162, 2005.

[39] S. P. Weisberg, R. Leibel, and D. V. Tortoriello, "Dietary curcumin significantly improves obesity-associated inflammation and diabetes in mouse models of diabesity," Endocrinology, vol. 149, no. 7, pp. 3549-3558, 2008.

[40] K. Reyes-Gordillo, J. Segoviab, M. Shibayama et al., "Curcumin prevents and reverses cirrhosis induced by bile duct obstruction or $\mathrm{CCl}_{4}$ in rats: role of TGF- $\beta$ modulation and oxidative stress," Fundamental and Clinical Pharmacology, vol. 22, no. 4, pp. 417427, 2008

[41] S. S. Yun, S. P. Kim, M. Y. Kang, and S. H. Nam, "Inhibitory effect of curcumin on liver injury in a murine model of endotoxemic shock," Biotechnology Letters, vol. 32, no. 2, pp. 209-214, 2010.

[42] F. Vizzutti, A. Provenzano, S. Galastri et al., "Curcumin limits the fibrogenic evolution of experimental steatohepatitis," Laboratory Investigation, vol. 90, no. 1, pp. 104-115, 2010.

[43] B. J. Kudchodkar, A. G. Lacko, L. Dory, and T. V. Fungwe, "Dietary fat modulates serum paraoxonase 1 activity in rats," Journal of Nutrition, vol. 130, no. 10, pp. 2427-2433, 2000.

[44] B. Fuhrman, N. Volkova, and M. Aviram, "Pomegranate juice polyphenols increase recombinant paraoxonase-1 binding to high-density lipoprotein: Studies in vitro and in diabetic patients," Nutrition, vol. 26, no. 4, pp. 359-366, 2010.

[45] J. Hamelet, K. Demuth, J. Dairou et al., "Effects of catechin on homocysteine metabolism in hyperhomocysteinemic mice," Biochemical and Biophysical Research Communications, vol. 355, no. 1, pp. 221-227, 2007.

[46] R. A. Sharma, C. R. Ireson, R. D. Verschoyle et al., "Effects of dietary curcumin on glutathione S-transferase and malondialdehyde-DNA adducts in rat liver and colon mucosa: relationship with drug levels," Clinical Cancer Research, vol. 7, no. 5, pp. 1452-1458, 2001.

[47] S. V. Singh, X. Hu, S. K. Srivastava et al., "Mechanism of inhibition of benzo[a]pyrene-induced forestomach cancer in mice by dietary curcumin," Carcinogenesis, vol. 19, no. 8, pp. 1357-1360, 1998. 


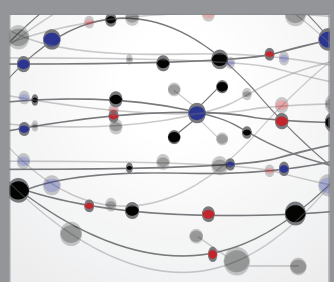

The Scientific World Journal
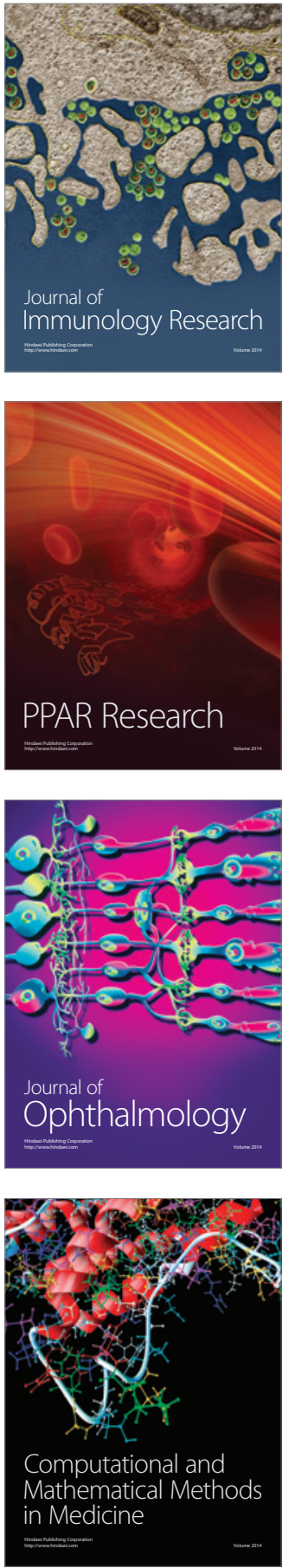

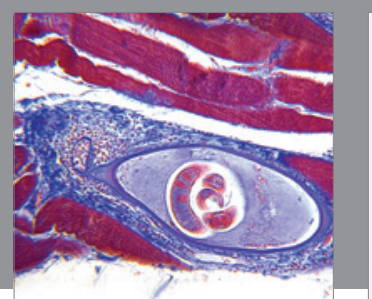

Gastroenterology Research and Practice

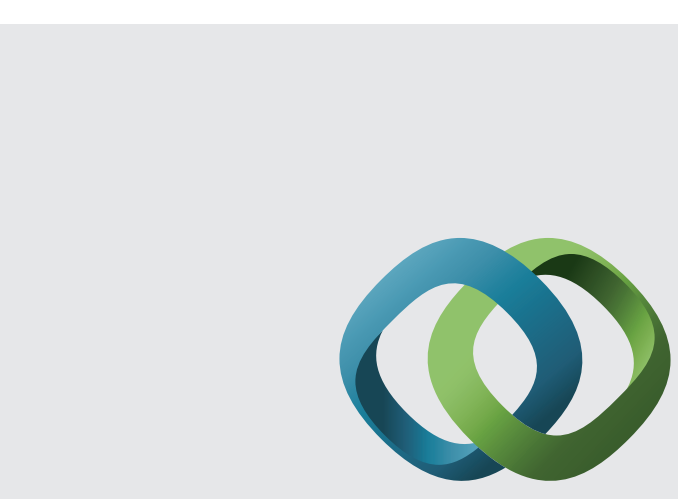

\section{Hindawi}

Submit your manuscripts at

http://www.hindawi.com
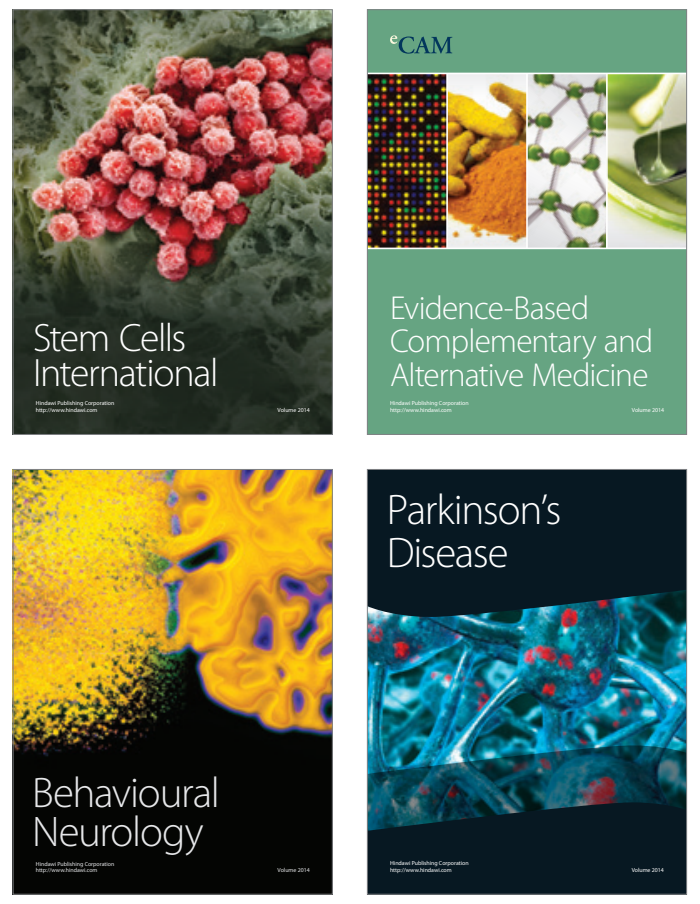
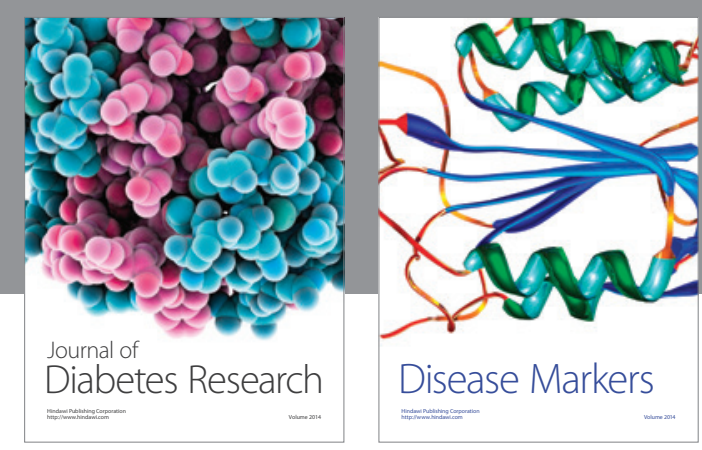

Disease Markers
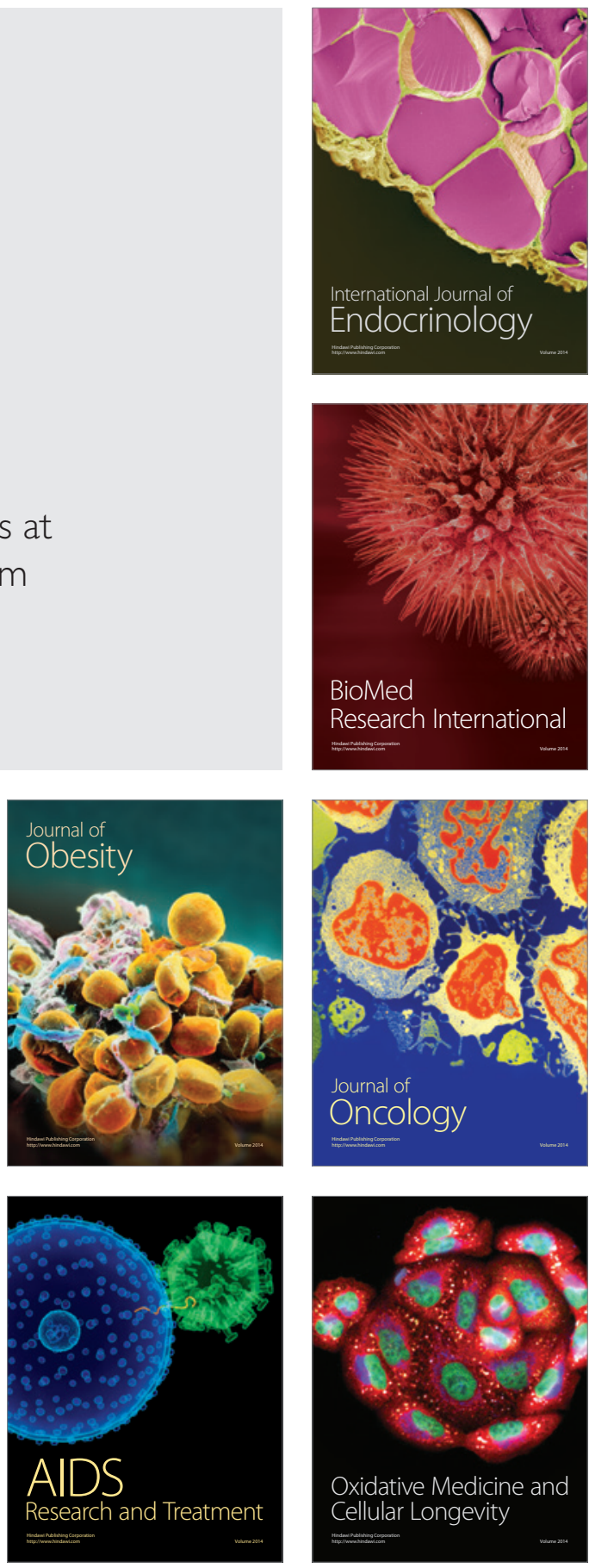